\title{
Control algebraico de una esfera suspendida magnéticamente: Diseño e implementación en hardware usando un procesador digital de señales
}

\author{
Algebraic control of a magnetically suspended sphere: Design and \\ hardware implementation using a digital signal processor \\ V. A. Ortiz-Bravo ; M. A. Nieto-Arias ; L. E. Avendaño-González iD ; J. P. \\ González-Tamayo iD
}

\begin{abstract}
Magnetic levitation is the phenomenon that occurs when an object is suspended in the air by the action of a magnetic field, either by repulsion or by attraction, with practical applications such as the MagLev train. The main objective is to design a control methodology that allows to improve the characteristics such as greater speed in response times, stability, robustness to external disturbances and in general greater efficiency in the production processes; for this, two control techniques were designed, one by pole relocation and the other with an algebraic control scheme for the comparison of the behavior of the system in terms of stability and robustness in presence of disturbances. The algebraic control method exhibits better performance compared to conventional methods, since it reduces oscillatory behavior and it has greater rejection to external disturbances. We concluded that the use of a high-speed processing device guaranteed that the algorithms executed in real time greatly improved the general response of the physical system.
\end{abstract}

Index Terms - Algebraic control; digital signal processor; instrumentation and control; magnetic levitation; pole relocation.

Resumen - La levitación magnética es el fenómeno que ocurre cuando un objeto se suspende en el aire por la acción de un campo magnético, ya sea por repulsión o por atracción, con aplicaciones prácticas como el tren MagLev. El objetivo principal es diseñar una metodología de control que permita mejorar características como mayor velocidad en tiempos de respuesta, estabilidad, robustez ante perturbaciones externas y en general

Este manuscrito fue enviado el 04 de agosto de 2019 y aceptado el 27 de febrero de 2020 .

V. A. Ortiz Bravo, Maestría en Instrumentación Física, Universidad Tecnológica de Pereira (e-mail: vialortiz@utp.edu.co)

M. A. Nieto Arias, Maestría en Instrumentación Física, Universidad Tecnológica de Pereira (e-mail: manieto@utp.edu.co)

L. E. Avendaño González, Tecnología Eléctrica, Universidad Tecnológica de Pereira (e-mail: leavenda@utp.edu.co)

J. P. González Tamayo, Facultad de Ingenierías, Universidad Tecnológica de Pereira (e-mail: jpgonzalez@utp.edu.co) una mayor eficiencia en los procesos productivos; para esto se diseñaron dos técnicas de control, una por reubicación de polos y la otra con un esquema de control algebraico para la comparación del comportamiento del sistema en cuanto a estabilidad y robustez ante perturbaciones. El método de control algebraico presenta un mejor desempeño en comparación con los métodos convencionales, ya que reduce el comportamiento oscilatorio y tiene mayor rechazo a perturbaciones externas. Se concluye que la utilización de un dispositivo de alta velocidad de procesamiento, garantizó que los algoritmos ejecutados en tiempo real mejoraran considerablemente la respuesta general del sistema físico.

Palabras claves - Control algebraico; instrumentación y control; levitación magnética; procesador digital de señales; reubicación de polos.

\section{INTRODUCCIÓN}

Ц A levitación magnética es el fenómeno que ocurre cuando un objeto se suspende en el aire por la acción de un campo magnético, ya sea por repulsión o por atracción [1]. La levitación así mencionada se efectúa sobre el elemento a suspender mediante un sistema que genera un campo magnético en el cual se sumerge dicho elemento, de forma que se generen fuerzas que se contraponen a la fuerza de atracción de la gravedad [2].

Las aplicaciones van desde la suspensión de pequeños cuerpos para uso en entretenimiento, pasando por la aplicación en trenes cuyo desplazamiento utiliza la levitación, hasta llegar a aplicaciones complejas en la fusión nuclear donde se suspende el plasma mediante campos magnéticos, dada la imposibilidad de contenerlo en materiales comunes [1]. Lo mencionado sugiere que es importante la aplicación de nuevos métodos de control en la investigación académica e industrial para optimizar ciertos procesos de automatización. De esta forma, se obtienen mejoras tales como: mayor velocidad en tiempos de respuesta, estabilidad, robustez ante perturbaciones externas y en general una mayor eficiencia en los procesos productivos [3][4][5]. 
Recientemente se han realizado investigaciones, en las que se han diseñado e implementado controladores difusos para realizar tareas de regulación y seguimiento en la dinámica no lineal de sistemas [6]. Técnicas como el regulador linealcuadrático (LQR) y el controlador de media móvil no lineal autorregresiva (NARMA) basado en redes neuronales [7], permitieron obtener resultados concluyentes en cuanto a las especificaciones de desempeño del sistema.

De otra parte, se han desarrollado sistemas de control lineal para este tipo de sistemas, a través de compensadores [8] y realimentación de estados con integrador [9], obteniendo respuestas satisfactorias en términos de error de estado estacionario y añadiendo un menor costo computacional, con respecto a la implementación de controladores óptimos y no lineales.

En el presente proyecto, se aplica el método de control algebraico para elaborar el proceso de control en la levitación de un cuerpo rígido [10].

El desarrollo tecnológico en sistemas de procesamiento digital de información, permite mayores velocidades en la implementación de los algoritmos de control. En el caso específico de este proyecto, se utiliza un procesador digital de señales como elemento generador del proceso de control; de esta forma, se reduce el tiempo de ejecución y respuesta para el esquema de control propuesto, posibilitándose el procesamiento en tiempo real [11].

El artículo está organizado como sigue. La implementación de la planta y el esquema de monitoreo de la misma se presentan en la Sección 2, donde se describen los elementos utilizados en la construcción, así como los actuadores y el acondicionamiento de las señales, finalizando en la construcción del modelo matemático. En la Sección 3 se describe la técnica de control algebraico, así como su implementación en el DSP, la validez se procura verificar en comparación con un control por reubicación de polos [11][12]. En la Sección 4 se realiza el análisis sobre los resultados y en la Sección 5 se plantean las conclusiones.

\section{MARCO TEÓRICO}

\section{A. Modelado e implementación}

El sistema levitador magnético en conjunto con el controlador y la instrumentación correspondiente, se ilustran en el diagrama de bloques de la Fig. 1. A continuación se explican los diferentes elementos del proyecto.

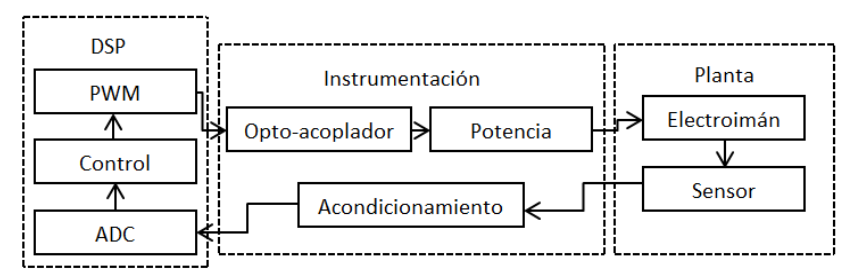

Fig. 1. Funcionamiento planteado para el control del sistema.

\section{1) Electroimán y cuerpo rígido}

El levitador está constituido por un solenoide con un núcleo de acero. El cuerpo rígido sometido a levitación es un cilindro de $3 \mathrm{~mm}$ de altura por $1.3 \mathrm{~cm}$ de diámetro. La Fig. 2 ilustra el sistema.

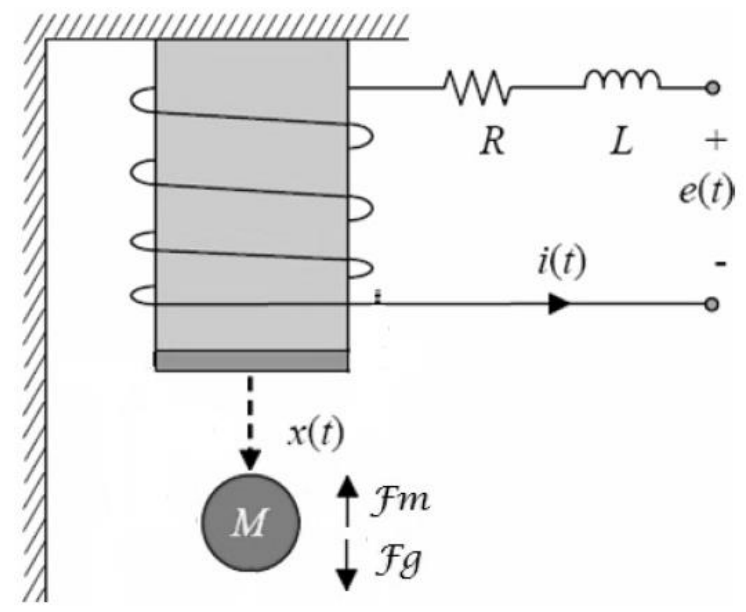

Fig. 2. Representación del sistema masa - bobina.

Del esquema mostrado en la Fig. 2 se pueden deducir las siguientes relaciones básicas para el modelo de la planta [13]. Aplicando la segunda ley de Kirchhoff al electroimán se obtiene:

$$
e(t)=R i(t)+L \frac{d i}{d t}
$$

Donde $e(t)$ es la tensión en los terminales del solenoide, la señal de excitación entregada por el controlador, $R$ es la resistencia interna del solenoide, $i(t)$ es la corriente por el mismo y $L$ es el valor de la inductancia del electroimán. Los parámetros $R$ y $L$ se consideran constantes, aunque su naturaleza es eventualmente variable. El diagrama de fuerzas sobre el objeto a levitar sometido, tanto a la fuerza de gravedad como a la influencia del campo magnético, está dado por (2):

$$
m \ddot{x}=F_{g}-F_{m}
$$

Donde $m$ es la masa del objeto a levitar, $x(t)$ es la distancia desde el centro de masa del objeto hasta la superficie del electroimán, $F_{m}$ y $F_{g}$ son las fuerzas magnéticas y gravitacionales que actúan sobre el objeto.

Aplicando transformaciones algebraicas y la transformada de Laplace a las ecuaciones resultantes, se obtiene la función de transferencia del sistema, donde la entrada es la tensión en el electroimán y la salida es la distancia del objeto con respecto al electroimán [14].

$$
H(s)=\frac{X(s)}{U(s)}=\frac{\frac{2}{x_{0} L} \sqrt{\frac{k g}{m}}}{\left(s+\frac{R}{L}\right)\left(s^{2}-\frac{2 g}{x_{0}}\right)}
$$

Como se puede observar en (3), la planta es de tercer orden, donde dos de los polos son estables situados en:

$$
S=-\frac{R}{L} \quad \text { y } S=-\sqrt{\frac{2 g}{x_{0}}}
$$


y uno inestable en:

$$
s=\sqrt{\frac{2 g}{x_{0}}}
$$

Pero por el criterio de polos dominantes, se puede deducir que la respuesta de este sistema no depende del polo $s=-R / L$, ya que se encuentra en $s=-211$ [14].

Para reducir la complejidad del modelo y considerando el análisis cerca de los polos dominantes, se suprime el polo más alejado; por lo que (3) se puede expresar como se observa en (4):

$$
H(s)=\frac{\frac{2}{x_{0} L} \sqrt{\frac{k g}{m}}}{s^{2}-\frac{2 g}{x_{0}}}
$$

Donde $x_{0}=0.0263 \mathrm{~m}$ es el punto de operación sobre el cual se linealiza el sistema, $m=2.53 g$ es la masa del objeto a levitar, $g$ es la aceleración de la gravedad e $i_{0}$ la corriente en el punto de operación.

El valor de la resistencia del electroimán fue medida por medio de un multímetro digital, obteniendo el valor de $R=$ $33.5 \Omega$. El valor de $L$ se obtuvo mediante el análisis asociado a un modelo $R L$ y usando un barrido en frecuencia. La frecuencia de corte está dada por:

$$
f_{c}=\frac{R}{2 \pi L}
$$

Así, conociendo a qué frecuencia la señal se atenúa en $3 \mathrm{~dB}$, de (5) se obtiene un valor de $L=84.13 \mathrm{mH}$ [5].

La constante $\mathrm{k}$ es propia del electroimán y se halla de manera empírica variando la posición del objeto levitante y midiendo el valor de la tensión y la corriente requerida por el electroimán, para que la fuerza magnética sea aproximadamente igual al peso del objeto. Después de calcular estos datos en el punto de operación, se obtiene su valor como:

$$
k=m g \frac{x_{0}^{2}}{i_{0}^{2}}
$$

Obteniendo un $k=0.000678366 \frac{\mathrm{kg} \cdot \mathrm{m}^{3}}{\mathrm{~A}^{2} \cdot \mathrm{s}^{2}}$

De (4) y reemplazando los valores de las constantes calculadas se obtiene (7):

$$
H(s)=\frac{-1465}{s^{2}-745,2}
$$

Que corresponde al modelo aproximado de la función de transferencia del sistema a controlar. Los polos correspondientes se ubican en el eje real con valores aproximados de 27,3 y -27,3, lo que evidencia la inestabilidad del sistema al poseer una raíz en el semiplano complejo derecho.

\section{2) Sensor de efecto Hall}

El sensor de efecto Hall permite establecer la posición en la cual se encuentra el objeto levitador, variando la tensión en sus terminales de manera proporcional al campo magnético generado por éste. Por esta razón, el cuerpo levitador debe tener un campo magnético permanente [14]. Para la caracterización del sensor se tomaron diferentes distancias alrededor del punto de operación con su respectiva tensión, y al aplicar una regresión polinomial de tercer orden se obtuvo la línea de tendencia, la cual está gobernada por (8) [15].

$$
d=0.1122 u^{3}-0.3308 u^{2}+0.3359 u-0.0886
$$

Donde $d$ es la distancia en metros del electroimán al objeto y $u$ es la tensión en voltios entregada por el sensor.

\section{3) Circuito de potencia}

Puesto que la señal entregada por el DSP tiene un rango de tensión entre $0 \mathrm{~V}$ y $3.3 \mathrm{~V}$ y una corriente entre $0 \mathrm{~mA}$ a $20 \mathrm{~mA}$, es necesario amplificar la señal de tensión hasta $10 \mathrm{~V}$ y la corriente hasta $800 \mathrm{~mA}$. Para este propósito se utilizó el circuito CI L298N, en el cual la entrada y la salida conservan la misma característica de frecuencia, pero con diferentes niveles de tensión y corriente.

\section{4) Opto-acoplador}

Debido a posibles sobre - corrientes y corrientes inversas que pueden ser generadas por el CI L298N, es necesario utilizar el circuito CI 4N36 que aísle eléctricamente las etapas de procesamiento y de potencia.

\section{5) Circuito de acondicionamiento}

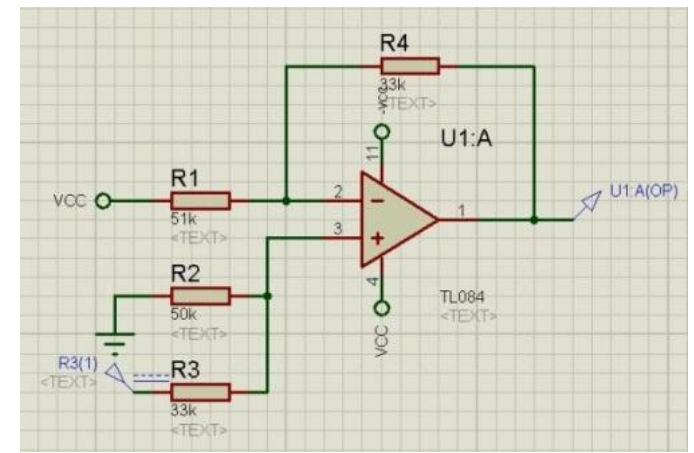

Fig. 3. Esquema del circuito de acondicionamiento utilizado.

Para el acople entre el sensor y el módulo conversor análogo digital del DSP, se llevó el rango de la tensión entregada por el sensor (que varía entre $3.77 \mathrm{~V}$ y $6.33 \mathrm{~V}$ ) a niveles que puedan ser utilizados por el DSP entre $0.5 \mathrm{~V}$ y 3.2V. Para la realización de este circuito se utilizó el amplificador operacional TL084 en la configuración sumadorrestador [15].

\section{6) Procesador digital de señales}

Para la etapa de procesamiento se usó un DSP de la Texas Instruments F2812, programado en el entorno de desarrollo Code Composer Studio (CCS) v. 3.1. A través del cual se adquiere la señal analógica entregada por el sensor, se digitaliza y se obtiene el valor correspondiente en distancia 
por medio de (7) [13]. Después de adquirir la posición del objeto, se diseñó un filtro pasa bajas digital de segundo orden con la siguiente función de transferencia:

$$
f(z)=\frac{0.04245 z+0.03659}{z^{2}-1.562 z+0.6413}
$$

El cual permite eliminar el ruido de alta frecuencia y el aliasing generado por el muestreo [15][16].

\section{B. Metodología de control}

En los procesos convencionales de control se utiliza una metodología de diseño conocida como enfoque hacia afuera, la cual consiste en primero elegir una configuración para el esquema de control, luego se calculan los parámetros, de modo que la función de transferencia global cumpla con las características específicas establecidas por el diseñador. Este enfoque es esencialmente un método de prueba y error; por lo tanto, se elige normalmente la configuración de realimentación lo más sencilla posible (es decir, configuración de realimentación unitaria) y se calcula el compensador más simple; es decir, una ganancia (un compensador de grado 0). Si el objetivo de diseño no se puede cumplir mediante la determinación de la ganancia, entonces se escoge una configuración diferente o un compensador de grado 1 (red de adelanto o de atraso de fase) y se repite el cálculo. Este enfoque parte de compensadores internos y luego se llega a un sistema general para cumplir las especificaciones de diseño; por esta razón se denomina enfoque hacia afuera.

En este artículo se aplica un procedimiento diferente denominado enfoque hacia adentro, en el que primero se busca una función de transferencia global que reúna las especificaciones de diseño, entonces se escoge una configuración de realimentación y se calculan los parámetros del compensador requerido; este esquema se denomina método de control algebraico. La función de transferencia global se puede encontrar aplicando algún método de optimización. El problema de diseño es encontrar una función de transferencia global para minimizar un índice de desempeño cuadrático con restricciones impuestas por requerimientos de diseño [3].

Para poner a prueba el diseño frente a los modelos convencionales, inicialmente se realizó el diseño de un sistema de control aplicando el método de reubicación de polos con el fin de comparar dicho diseño con el método algebraico y de esta manera obtener datos relevantes en cuanto a la respuesta del sistema, esfuerzo de la señal de control, estabilidad y rechazo a perturbaciones.

\section{METODOLOGÍA}

\section{A. Control por reubicación de polos}

Para la aplicación de esta metodología de control, es necesario conocer el modelo de la planta y la ecuación característica (en este caso de segundo orden), el tiempo de establecimiento y el sobreimpulso observado. Con estos elementos se obtienen las constantes que determinan el comportamiento en lazo cerrado del sistema [18]. El diseño se desarrolla utilizando un procedimiento en el dominio discreto, como se muestra a continuación.

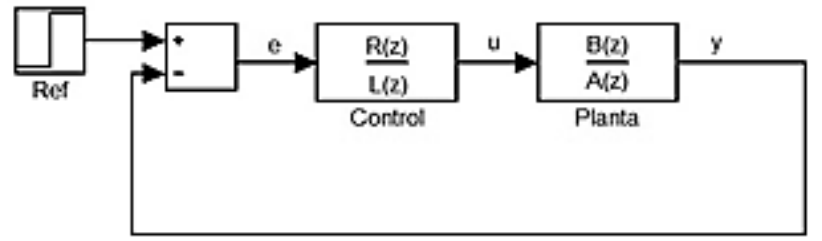

Fig. 4. Esquema general de control por reubicación de polos.

\section{B. Diseño del algoritmo de control}

La función de transferencia de la planta se describe a continuación:

$$
H(z)=\frac{B(z)}{A(z)}=\frac{b_{1} z+b_{0}}{z^{2}+a_{1} z+a_{0}}
$$

La función de transferencia del controlador está dada por la expresión:

$$
G(z)=\frac{R(z)}{L(z)}=\frac{r_{1} z+r_{0}}{z-1}
$$

La función de transferencia en lazo cerrado se expresaría como:

$$
H_{l c}(z)=\frac{R(z) B(z)}{L(z) A(z)+R(z) B(z)}
$$

Planteando las expresiones anteriores en forma matricial, se obtiene el siguiente sistema de ecuaciones [14].

$$
\left[\begin{array}{ccc}
0 & b_{1} & -1 \\
b_{1} & b_{0} & -a_{1} \\
b_{0} & 0 & -a_{0}
\end{array}\right]\left[\begin{array}{c}
r_{0} \\
r_{1} \\
l
\end{array}\right]=\left[\begin{array}{c}
\alpha_{1}+a_{2} \\
\alpha_{2}-a_{2} \\
\alpha_{3}
\end{array}\right]
$$

Donde $\alpha_{1}, \alpha_{2} y \alpha_{3}$ son los coeficientes del denominador deseado. Resolviendo este sistema se obtienen las constantes del controlador.

$$
\begin{gathered}
r_{0}=626.360 \\
r_{1}=-700.046 \\
l=0.2283
\end{gathered}
$$

\section{Control algebraico}

El control algebraico tiene diferentes tipos de configuraciones, para este caso se usó el esquema de control denominado configuración de dos parámetros, el cual se puede implementar sobre cualquier función de transferencia realizable [3] y se define de acuerdo a la Figura 5.

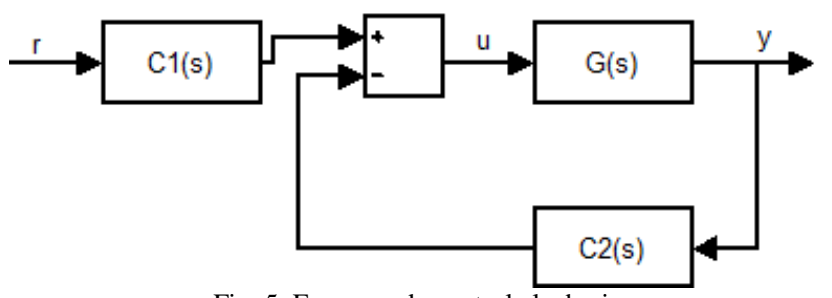

Fig. 5. Esquema de control algebraico.

$$
C_{1}(s)=\frac{L(s)}{A(s)} ; C_{2}(s)=\frac{M(s)}{A(s)}
$$


Donde:

$$
\begin{gathered}
A(s)=A_{0}+A_{1} s+A_{2} s^{2}+\cdots .+A_{m} s^{m} \\
M(s)=M_{0}+M_{1} s+M_{2} s^{2}+\cdots \cdot+M_{m} s^{m}
\end{gathered}
$$

En (15) $C_{l}(s)$ y $C_{2}(s)$ son los componentes del vector compensador, los cuales se encargan respectivamente de rastrear la referencia y estabilizar el sistema [3].

\section{Diseño algoritmo de control}

Se tiene la función de transferencia de la forma:

Donde:

$$
G(s)=\frac{N(s)}{D(s)}
$$

$$
\begin{gathered}
D(s)=D_{0}+D_{1} s+D_{2} s^{2}+\cdots+D_{n} s^{n} \quad D_{n} \neq 0 \\
N(s)=N_{0}+N_{1} s+N_{2} s^{2}+\cdots+N_{n} s^{n}
\end{gathered}
$$

El problema de diseño es hallar una función de transferencia global que permita minimizar el índice de desempeño cuadrático definido por la expresión:

$$
J=\int_{0}^{\infty}\left\{q[y(t)-r(t)]^{2}+u^{2}(t)\right\} d t
$$

Donde $q$ es una constante positiva, $r(t)$ es la señal de referencia, $y(t)$ es la variable de salida y $u(t)$ es la señal que establece la ley de control. Dentro de la integral en (17) se tiene el error de rastreo, lo cual permite que en la implementación no solamente el cuerpo rígido pueda mantenerse en una posición determinada, sino que pueda hacer seguimiento a una trayectoria predefinida. Así mismo, se reduce el esfuerzo en la señal de control $u(t)$.

La función de transferencia global según la cual se debe comportar el sistema en lazo cerrado, se define así:

$$
G_{0}(s)=\frac{N_{0}(s)}{D_{0}(s)}=\frac{q N(0)}{D_{0}(0)} \frac{N(s)}{D_{0}(s)}
$$

Ahora se procede a realizar la implementación de la función de transferencia global. Es decir, hay que hallar los compensadores necesarios para que $G(s)$ se transforme en $G_{0}(s)$. Puesto que el grado de $\partial D_{0}(s)=n+m \partial D_{0}(s)=n+$ $m$, donde el símbolo $\partial \partial$, indica el grado del polinomio, entonces $D_{0}(s)$ se puede expresar como:

$$
D_{0}(s)=F_{0}+F_{1} s+F_{2} s^{2}+\cdots+F_{n+m} s^{n+m}
$$

Esto conduce a la representación matricial que se muestra a continuación:

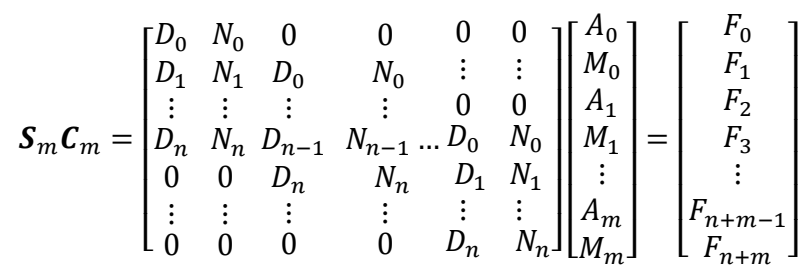

En (20) se tiene una solución para cualquier $F_{i}$, o equivalentemente para cualquier $D_{0}(s)$ si y solo si la matriz $S_{m}$ tiene una fila de rango completo.

Para poder implementar este método de control en el procesador digital de señales, se realiza la discretización de la función de transferencia global y de la función de transferencia de la planta. En este caso se ha utilizado un retenedor de orden cero.

$$
\begin{gathered}
G(z)=\frac{N(z)}{D(z)}=\frac{N_{1} z+N_{0}}{D_{2} z^{2}+D_{1} z+D_{0}} \\
G_{0}(z)=\frac{N_{0}(z)}{D_{0}(z)}=\frac{q N(0)}{D_{0}(0)} \frac{N(z)}{D_{0}(z)}
\end{gathered}
$$

Se introduce un polinomio Hurwitz aleatorio $\widehat{D} p(z)$; sin embargo, debe ser un polinomio con polos aleatorios estables.

$$
\widehat{D}_{p}(z)=z+\beta_{0}
$$

Ahora se escribe la ecuación Diofántica de la siguiente manera:

$$
\left[\begin{array}{cccc}
D_{0} & N_{0} & 0 & 0 \\
D_{1} & N_{1} & D_{0} & N_{0} \\
D_{2} & N_{2} & D_{1} & N_{1} \\
0 & 0 & D_{2} & N_{2}
\end{array}\right]\left[\begin{array}{l}
A_{0} \\
M_{0} \\
A_{1} \\
M_{1}
\end{array}\right]=\left[\begin{array}{c}
F_{0} \\
F_{1} \\
F_{2} \\
F_{3}
\end{array}\right]
$$

La ecuación Diofántica para este caso, está definida por los polinomios:

$$
\begin{gathered}
A(z)=A_{0}+A_{1} z, \\
M(z)=M_{0}+M_{1} z, \\
L(z)=N_{0}(z) \widehat{D} p(z) \\
C_{1}(z)=\frac{L(z)}{A(z)} ; \quad C_{2}(z)=\frac{M(z)}{A(z)}
\end{gathered}
$$

\section{RESULTADOS}

\section{A. Control por reubicación de polos}

De acuerdo con (12) y a los resultados obtenidos en (14), se efectúa la simulación en $\mathrm{Matlab}^{\circledR}$ y posteriormente se implementa el algoritmo de control en el DSP con el fin de obtener la respuesta del sistema en tiempo real.

En la Fig. 6 se observa el comportamiento de la señal de control obtenida por un método convencional basado en el error entre la referencia y la salida. Las cotas tanto superior como inferior se encuentran dentro de un rango factible para el actuador usado. Además, se muestra el efecto de una perturbación en la muestra número 100 , generando un sobre impulso de $1.8 \mathrm{~V}$ y un sub impulso de $-0.5 \mathrm{~V}$. A partir de la muestra número 115 el sistema retorna a su punto de operación. 


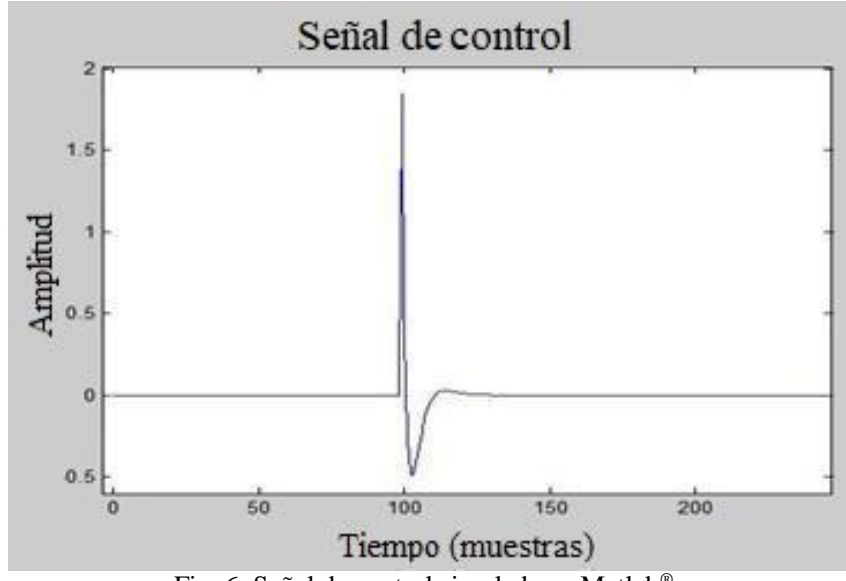

Fig. 6. Señal de control simulada en Matlab ${ }^{\circledR}$.

En la Fig. 7 se observa la salida controlada del sistema simulado, la cual tiene un sobre impulso considerable y una estabilización en menos de 30 muestras.

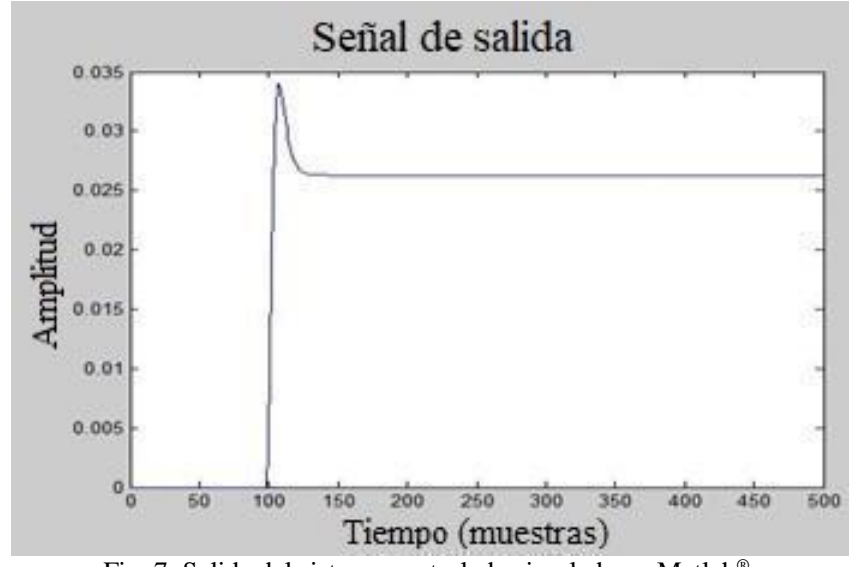

Fig. 7. Salida del sistema controlado simulado en Matlab ${ }^{\circledR}$.

Ahora se aplica el mismo procedimiento al sistema físico. En la Fig. 8 se puede apreciar que la señal de control posee transiciones positivas y negativas, con el fin de mantener el objeto levitante en una posición determinada.

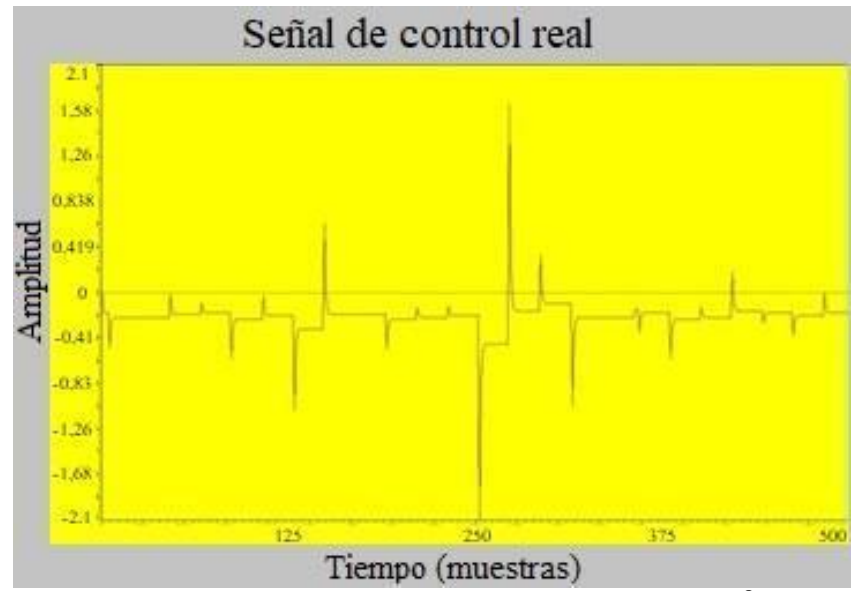

Fig. 8. Señal de control generada en el graficador de CCS $^{\circledR}$.

La señal de la Fig. 9 corresponde a la salida del sistema en tiempo real. Se observa que tiene un leve error en estado estacionario $\mathrm{y}$ posee desviaciones positivas $\mathrm{y}$ negativas alrededor del punto de operación, debido a las vibraciones en el objeto levitante.

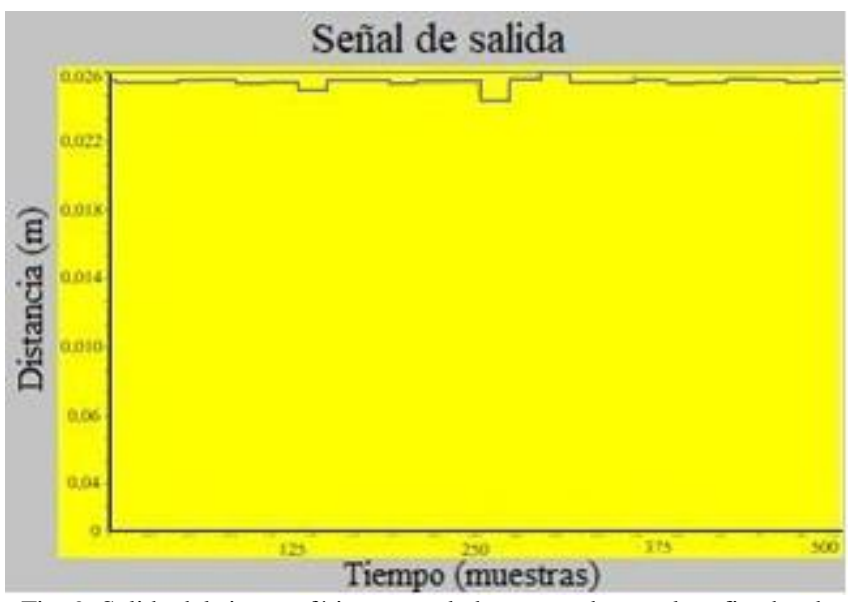

Fig. 9. Salida del sistema físico controlado, generada por el graficador de $\mathrm{CCS}^{\circledR}$.

\section{B. Control Algebraico}

En la Fig. 10 se observa que los límites de la señal de control simulada se encuentran dentro del rango esperado.

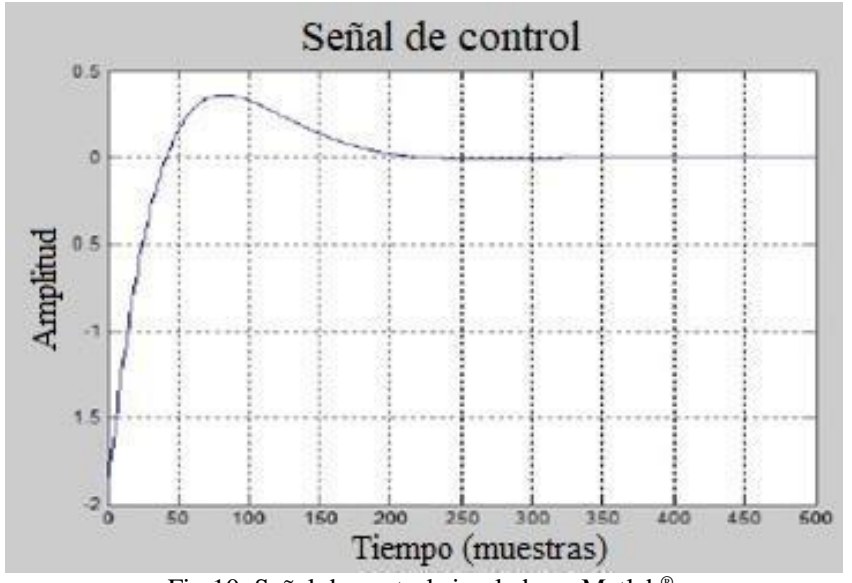

Fig 10. Señal de control simulada en Matlab ${ }^{\circledR}$.

En la Fig. 11 se observa que el rango en el que se está generando la señal de control es del orden de los milivoltios; es decir, aproximadamente 100 veces menos que la obtenida por la técnica de reubicación de polos de la Figura 8, lo que comprueba que el control por el método algebraico reduce el esfuerzo en la acción de control. 


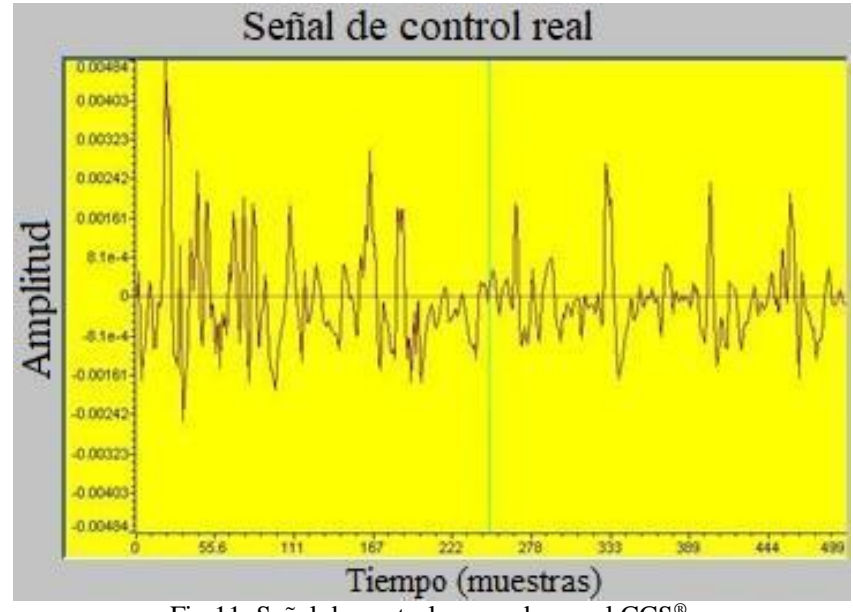

Fig 11. Señal de control generada por el $\mathrm{CCS}^{\circledR}$.

En la Fig. 12 se observa en la respuesta del sistema controlado, un leve sobreimpulso, un tiempo de establecimiento alrededor de 250 muestras y un error de estado estacionario nulo.

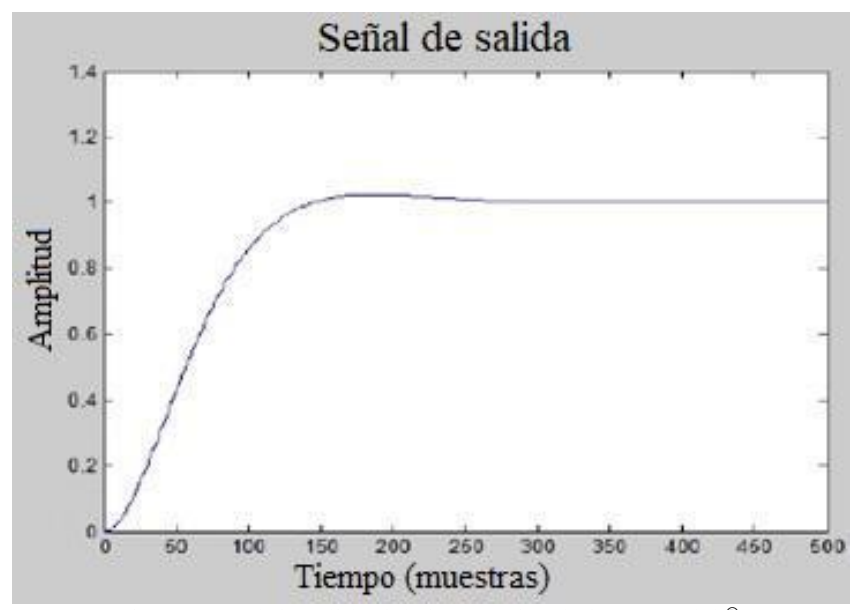

Fig 12. Señal de salida del sistema simulada en Matlab ${ }^{\circledR}$.

En la Fig. 13 se observa la salida del sistema físico controlado, que evidencia la estabilidad en la posición del objeto levitante, en comparación con la respuesta del controlador por reubicación de polos de la Figura 9.

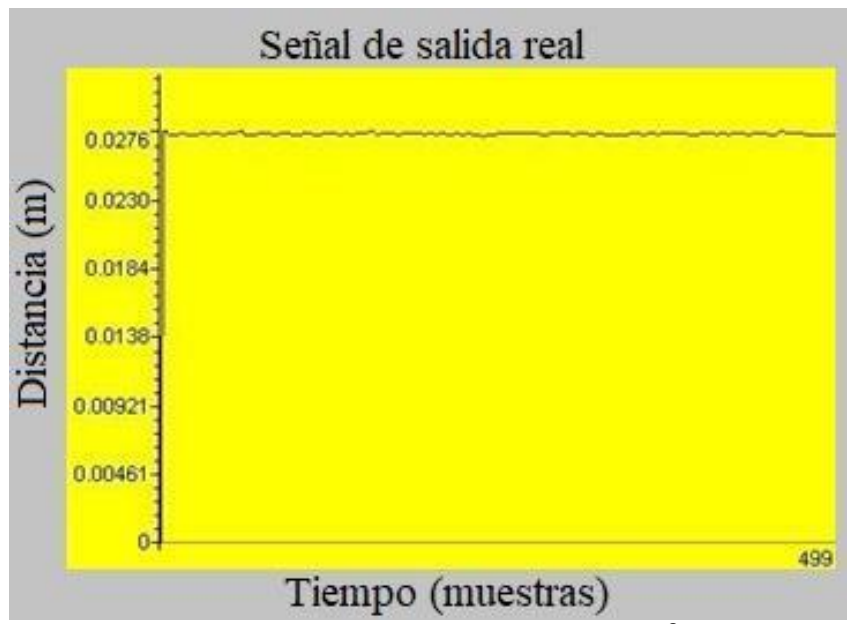

Fig. 13. Señal de salida generada por el $\mathrm{CCS}^{\circledR}$.

\section{DISCUSIÓN}

El control del sistema por reubicación de polos y el método algebraico funcionaron de la manera esperada, ya que ambos lograron hacer levitar magnéticamente el objeto y mostraron en los resultados gran similitud a los obtenidos en las simulaciones, aunque para el control por reubicación de polos fue necesaria la utilización de una guía vertical, de modo que el objeto no se alejara de la referencia. Adicionalmente y en comparación con investigaciones relacionadas en los últimos años, se puede concluir que, aunque existen múltiples metodologías de control para la estabilización del sistema, estos se basan en resultados simulados para la obtención de las respuestas tanto de control como de salida [7] [8] [9], lo que implica una idealización de los procesos tanto del sistema físico, del actuador y de la instrumentación utilizada.

Con respecto a las diferencias más significativas de los resultados obtenidos en esta investigación, el control por reubicación de polos genera vibraciones considerables en el objeto levitante como se observa en la Fig. 9 y transiciones abruptas de la señal de control (ver Fig. 8), mientras que el método algebraico reduce el esfuerzo de la señal de control como se observa en la Fig. 11; produciendo un comportamiento más estable y robusto a las perturbaciones (ver Fig. 13). Sin embargo, al ser un sistema no lineal, linealizado alrededor de un punto de operación, obliga a que por fuera de este valor la metodología de control implementada no funcione adecuadamente.

La implementación en el procesador digital de señales (DSP), permitió una ejecución correcta del algoritmo de control algebraico en tiempo real. Esta técnica requiere altas velocidades de procesamiento, puesto que el modelado matemático tanto de la planta de levitación como la metodología de control se basa esencialmente en procesos matriciales, lo que dificulta su implementación en sistemas embebidos de bajo rendimiento [17].

Finalmente es importante mencionar que como trabajo futuro se plantea la implementación de este sistema en dispositivos embebidos de menor rendimiento, optimizando el análisis matricial para generar una reducción en el costo computacional.

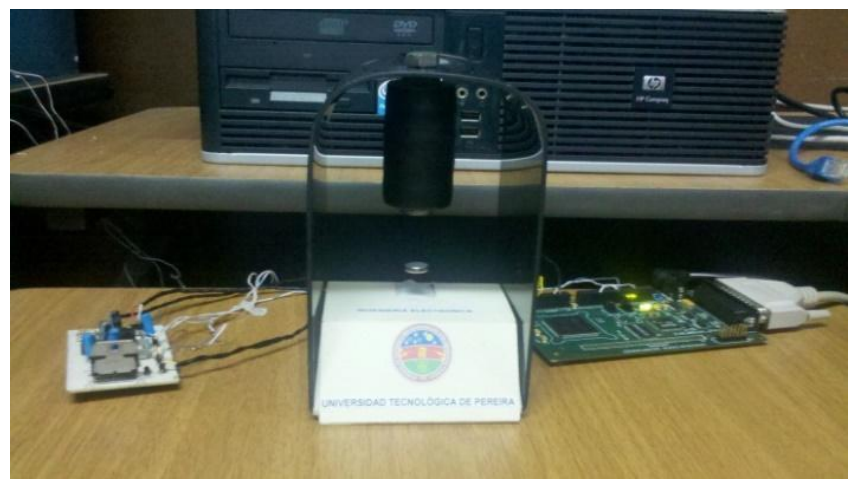

Fig. 14. Sistema final en funcionamiento. 


\section{REFERENCIAS}

[1] G. Perren, "Estudio de las aplicaciones prácticas de la levitación magnética (trenes maglev)”, Física 3, 2003.

[2] D. Rairán, "Levitación magnética: alternativa para el transporte de pasajeros", Universidad Distrital Francisco José de Caldas, 1a ed., Bogotá, Colombia, 2003.

[3] C.-T. Chen, "Analog and digital control system design: transferfunction, state-space, and algebraic methods", Saunders College Pub., 1993.

[4] F. Rodriguez and M. J. López, "Control adaptativo y robusto", Universidad de Sevilla, vol. 9, 1996.

[5] K. Ogata, "Modern control engineering", Fifth ed., Prentice Hall, NJ, 2010.

[6] R. Y. Almazan, O. O. Gutierrez, Y. Lozano y M. Villafuerte, "Control PI difuso de un sistema de levitación magnética mediante un sistema embebido", Ingeniería investigación y tecnología, vol. $\mathrm{xx}$, no. 4, pp. 1-11, Oct. 2019. DOI: 10.22201/fi.25940732e.2019.20n4.043

[7] E. P. Herrera, K. A. Herrera, I. D. Herrera, L. M. Sierra and D. H. Peluffo, "Comparación de controladores y modelado matemático de un levitador magnético", Revista Ibérica de Sistemas $e$ Tecnologias de Informação, no. E25, pp. 424-438, Ene. 2020.

[8] A. Angulo y J. G. Aviña, "Sistema de control digital para levitador magnético", Jóvenes en la ciencia: revista de divulgación científica, vol. 2, no. 1, pp. 1811-1816, 2016.

[9] J. S. Castro, C. F. Vergara y J. S. Herrera, "Comparación de un control lineal y no lineal en un levitador magnético", Ingenierías USBMed, vol. 9, no. 1, pp. 112-118, Ene. 2018.

[10] V. A. Ortiz y M. A. Nieto, "Control algebraico para un sistema de levitación magnética usando estimación de parámetros en tiempo real implementado en un procesador digital de señales", Tesis de pregrado, Programa de Ingeniería Electrónica, Universidad Tecnológica de Pereira, Pereira, Colombia, 2012.

[11] P. A. Laplante and S. J. Ovaska, "Real-time systems design and analysis: tools for the practitioner", Fourth ed., NJ, USA: John Wiley and Sons, 2011.

[12] V. Bobál, J. Böhm, J. Fessl, and J. Machácek, "Digital selftuning controllers: algorithms, implementation and applications", Springer, 2005.

[13] R. A. Serway and J. W. Jewett, "Física: Electricidad y magnetismo", 9a ed., Cengage, 2016.

[14] R. Pallas, "Sensores y Acondicionadores de Señal", 4a ed., Marcombo, 2005.

[15] L. E. Avendaño, "Fundamentos de instrumentación", Universidad Tecnológica de Pereira, 2002.

[16] L. E. Avendaño, "Sistemas Electrónicos Analógicos: Un Enfoque Matricial", Universidad Tecnológica de Pereira, 2006.

[17] S. K. Mitra, "Digital signal processing: a computer-based approach", 4a ed., McGraw-Hill, 2013.

[18] N. C. Cheung, Z. G. Sun, S. W. Zhao, and W.-C. Gan, "Design and simulation of a testing fixture for planar magnetic levitation system control using switched reluctance actuator", in 3rd International conference on power electronics systems and applications (PESA), IEEE, Hong Kong, China, 2009, pp. 1-6.

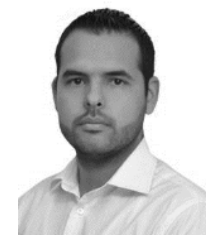

Víctor Alfonso Ortiz Bravo, nació en Pereira, Colombia en 1989. Obtuvo su título en Ingeniería Electrónica en el año 2012 en la Universidad Tecnológica de Pereira, Especialista en Edumática y Magíster en Pedagogía en el año 2017 en la Universidad Católica de Pereira, aspirante al grado de Magister en Instrumentación Física de la Universidad Tecnológica de Pereira, Profesor-Investigador con 6 años de experiencia, apasionado de las ciencias exactas, la educación y la investigación, especialmente en el campo de la instrumentación y el control, la bioingeniería y la ciencia de datos. Miembro de los grupos de investigación Applied Neuroscience y GIADSc.

ORCID: https://orcid.org/0000-0002-5574-4280

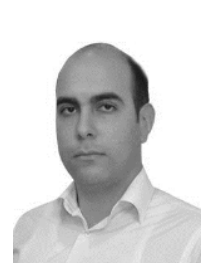

Manuel Antonio Nieto Arias, nació en Pereira, Colombia en 1990. Obtuvo su título en Ingeniería Electrónica en el año 2012 en la Universidad Tecnológica de Pereira, Especialista en Edumática y Magister en Pedagogía en el año 2017 en la Universidad Católica de Pereira, aspirante al grado de Magister en Instrumentación Física de la Universidad Tecnológica de Pereira.

ORCID: https://orcid.org/0000-0002-0633-4779

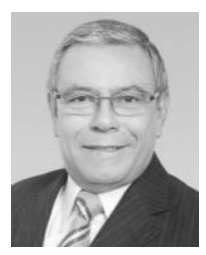

Luis Enrique Avendaño González, Ingeniero Electrónico de la Universidad del Cauca, Magister en Ingeniería Eléctrica de la Universidad de los Andes y Ph.D en Bioelectrónica de la Universidad de Valencia, Profesor e Investigador de la Universidad Tecnológica de Pereira. Áreas de interés: instrumentación y control, procesamiento de señales, ingeniería eléctrica y electrónica.

ORCID: https://orcid.org/0000-0002-2892-4382

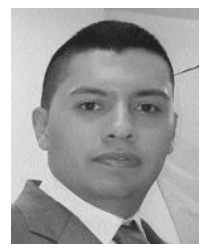

Juan Pablo González Tamayo, nació en Pereira, Colombia en 1981. Obtuvo su grado en Ingeniería Electrónica y maestría en Automatización Industrial en el año 2004 y 2006 respectivamente, en la Universidad Nacional de Colombia sede Manizales; actualmente es candidato a Doctor en Ciencias de la Educación en la Universidad Cuauhtémoc plantel Aguascalientes México. Desde el año 2006 hasta la actualidad, él ha sido docente e investigador del programa de Ingeniería Electrónica de la Universidad Tecnológica de Pereira. Ha publicado diferentes artículos relacionados con la implementación de estrategias de control moderno, lo que ha correspondido a su tema de interés en investigación.

ORCID: https://orcid.org/0000-0002-9676-4579 